\title{
POLYNOMIAL IDENTITIES FOR SIMPLE LIE SUPERALGEBRAS
}

\author{
M. D. GOULD 1
}

(Received 19 April, 1985; revised 25 February 1986)

\begin{abstract}
Polynomial identities for the generators of a simple basic classical Lie superalgebra are derived in arbitrary representations generated by a maximal (or minimal) weight vector. The infinitesimal characters occurring in the tensor product of two finite dimensional irreducible representations are also determined.
\end{abstract}

\section{Introduction}

Characteristic identities satisfied by the generators of a semi-simple Lie algebra have previously been obtained both in finite $[1,12]$ and infinite $[11,19]$ dimensional representations. Such polynomial identities have been shown to be a powerful tool for the analysis of finite dimensional representations and in particular are useful for projecting out shift components of tensor operators $[1,6,12]$. This latter application has recently been extended to infinite dimensional representations of a semi-simple Lie algebra [10]. Characteristic identity techniques are also useful for the explicit determination of the Wigner coefficients of a semi-simple Lie algebra [6,7]. More recently such techniques have been applied to the many electron problems of atomic physics and quantum chemistry $[8,9]$.

In this paper we determine the polynomial identities satisfied by the generators of a general simple (basic classical) Lie superalgebra. Much of the necessary formal mathematical machinery for investigating Lie superalgebras has now been developed primarily by Kac [16,18] and Scheunert et. al. [20,23, 24]. Lie superalgebras initially arose in physical applications in the context of elementary particle

\footnotetext{
${ }^{1}$ Present Address: School of Chemistry, The University of Western Australia, Nedlands, Western Australia, 6009, Australia.

(c) Copyright Australian Mathematical Society 1987, Serial-fee code 0334-2700/87
} 
physics and Fermi-Bose supersymmetry [5,21,22, 25]. A review of the various physical applications of Lie superalgebras is given in the paper by Corwin, Ne'eman and Sternberg [2].

This paper is motivated by the work of Jarvis and Green [14] who have determined the vector polynomial identities, in finite dimensional irreducible representations, for the general linear and ortho-symplectic Lie superalgebras. It is our aim here to generalize these results to obtain all tensor identities for a general basic classical Lie superalgebra. Our main reason for restricting ourselves to basic classical Lie superalgebras is that their root systems have been completely classified by Kac [18] and they possess a universal Casimir element in direct analogy with the normal semi-simple Lie algebra case. However, it is clearly evident that the approach of this paper extends to more general (simple) Lie superalgebras. We remark in this connection that Jarvis and Murray [15] have investigated characteristic identities satisfied by the "strange" Lie superalgebras $P(n)$ and $Q(n)$. These latter Lie superalgebras are simple, although not basic classical, and it is clear that our methods will extend to these algebras.

From the point of view of applications we remark that the polynomial identities derived in this paper are useful for the construction of projection operators which project out generalized shift components of super tensor operators along the lines suggested in [10]. Such methods are useful for an explicit determination of matrix elements of tensor operators which includes the important problem of obtaining the matrix elements of the Lie superalgebra generators in irreducible representations.

The paper is set up as follows. Our notation and basic conventions are established in Section 2. In Section 3 we derive the characteristic identities, satisfied by the generators of a basic classical Lie super-algebra on arbitrary representations (finite or infinite dimensional) generated by a maximal weight vector. As a by-product we determine the infinitesimal characters occurring in the tensor product of two irreducible representations. To clarify the connection with our results and those of Jarvis and Green [14] we consider in Section 4 the adjoint tensor identities. We conclude, in Section 5 , by investigating various generalizations. We remark that although we shall only consider the characteristic identities for representations generated by a highest weight vector it is clear that our results also hold for representations generated by a lowest weight vector.

\section{Notation and fundamentals}

Our notation follows mainly that of Humphreys [13] and Kac [18]. We assume that

$$
L=L_{0} \oplus L_{1}
$$


is a basic classical Lie superalgebra with even (resp. odd) part $L_{0}$ (resp. $L_{1}$ ): that is $L$ is either a simple Lie algebra or one of the classical Lie superalgebras in the following list

$$
A(m, n), B(m, n), C(n), D(m, n), D(2,1 ; \alpha), F(4), G(3) \text {. }
$$

We call $x \in L$ an even (resp. odd) element of $L$ if $x \in L_{0}$ (resp. $L_{1}$ ) in which case $x$ is said to be homogeneous of degree 0 (resp. 1 ). We denote the degree of a homogeneous element $x \in L$ by $(x)$ : we shall always assume, unless otherwise stated, that $x \in L$ is homogeneous. Now let

$$
U=U_{0} \oplus U_{1}
$$

denote the universal enveloping algebra of $L$ which constitutes a $\mathbf{Z}_{2}$-graded associative Noetherian algebra with even (resp. odd) part $U_{0}$ (resp. $U_{1}$ ). Without loss of generality we assume that $L$ is imbedded in $U$ in which case the graded bracket operation in $L$ may be written

$$
[x, y]=x y-(-1)^{(x)(y)} y x, \quad x, y \in L
$$

where $x y$ is shorthand notation for $x \otimes y$ (enveloping algebra product).

A bilinear form (,) on a Lie superalgebra $L$ is called invariant if it satisfies the following three conditions:

$$
\begin{array}{ll}
\text { (ii) } & (x, y)=0, \quad(x) \neq(y) \\
\text { (iii) } & ([x, y], w)=(x,[y, w]) .
\end{array}
$$

Following Kac [18] a basic classical Lie superalgebra $L$ is defined by the conditions that (a) $L$ is a simple Lie superalgebra [23], (b) the Lie algebra $L_{0}$ is reductive $[3,23]$ and (c) there exists a non-degenerate invariant bilinear form (,) on $L$.

Throughout this paper we let (,) denote a fixed non-degenerate invariant bilinear form on $L$. In view of Schur's lemma [20,24], we note that all invariant bilinear forms on $L$ must be a scalar multiple of the form (,). In particular the Killing form [20,23] of $L$ is a scalar multiple (possibly zero) of (,).

Now let $H$ be a fixed Cartan subalgebra (C.S.A.) of the even part $L_{0}$, herein referred to as the C.S.A. of $L$. We let $\Phi=\Phi_{0} \cup \Phi_{1}$ denote the set of roots of $L$ relative to $H$ with $\Phi_{0}$ (resp. $\Phi_{1}$ ) the set of even (resp. odd) roots. For $\alpha \in \Phi$ we let $L_{\alpha} \subset L$ denote the corresponding root space of $L$ : we have [18] $\operatorname{dim} L_{\alpha}=1$ for $L \neq A(1,1)$.

Let $B_{0}$ be the Borel subalgebra of $L_{0}$ generated by the C.S.A. $H$ and the positive root space vectors $x_{\alpha} \in L_{0}(\alpha>0)$ and let

$$
B=B_{0} \oplus B_{1}
$$


be a fixed Borel subalgebra of $L$. We obtain the following decomposition of $L$ :

$$
L=N^{-} \oplus H \oplus N^{+}, \quad B=H \oplus N^{+}
$$

where $N^{-}$and $N^{+}$are nilpotent subalgebras of $L$ and $\left[H, N^{ \pm}\right] \subseteq N^{ \pm}$.

A root $\alpha \in \Phi$ is called positive (resp. negative) if $L_{\alpha} \subseteq N^{+}$(resp. $N^{-}$). We denote the set of positive roots of $L$ by $\Phi^{+}$and we let $\Phi_{0}^{+}$(resp. $\Phi_{1}^{+}$) denote the subset of even (resp. odd) positive roots. We similarly define the negative root systems $\Phi^{-}=-\Phi^{+}, \Phi_{0}^{-}=-\Phi_{0}^{+}$and $\Phi_{1}^{-}=-\Phi_{1}^{+}$: we have [18] $\Phi=\Phi^{+} \cup \Phi^{-}$, $\Phi_{0}=\Phi_{0}^{+} \cup \Phi_{0}^{-}$and $\Phi_{1}=\Phi_{1}^{+} \cup \Phi_{1}^{-}$. We let $\delta_{0}$ (resp. $\delta_{1}$ ) denote the half-sum of the even (resp. odd) positive roots and we set

$$
\delta=\delta_{0}-\delta_{1}
$$

herein referred to as the graded half-sum of positive roots. Finally, we let > denote the partial ordering induced on the C.S.A. dual $H^{*}$ by the positive roots: that is $\lambda>\mu$ if and only if $\lambda-\mu$ is a positive $Z$-linear combination of positive roots $\left(\lambda, \mu \in H^{*}\right)$.

Following Kac [18] the invariant bilinear form (,) when restricted to $H$ is non-degenerate and hence induces a symmetric non-degenerate bilinear form on $H^{*}$ defined by

$$
(\mu, \lambda)=(\lambda, \mu)=\left(h_{\lambda}, h_{\mu}\right) ; \quad \lambda, \mu \in H^{*}
$$

where $h_{\lambda}$ is uniquely determined by

$$
\left(h_{\lambda}, h\right)=\lambda(h), \quad h \in H .
$$

A more detailed discussion of Lie superalgebras and their root systems (including a list of root systems for all the basic classical Lie superalgebras) is given in Kac [18].

Now let $V$ be a $U$-module: throughout we assume that all $U$-modules are graded. We let $V_{\lambda} \subseteq V\left(\lambda \in H^{*}\right)$ denote the subspace spanned by vectors of weight $\lambda$; viz.

$$
V_{\lambda}=\{v \in V \mid h v=\lambda(h) v, \forall h \in H\} .
$$

In such a case the weight $\lambda$ is said to occur with multiplicity $\operatorname{dim} V_{\lambda}$ in $V$. We note, since the Cartan generators are even, that if $v \in V_{\lambda}$ then the even and odd parts of $v$ also belong to $V_{\lambda}$. A vector $v_{0} \in V_{\lambda}$ is called a maximal weight vector of weight $\lambda$ if

$$
N^{+} v_{0}=0 .
$$

Following Humphreys [13] let us agree to call a $U$-module (finite or infinite dimensional) cyclically generated by a maximal weight vector of weight $\lambda \in H^{*}$ a standard cyclic module of highest weight $\lambda$. In such a case we may always regard the highest weight vector as homogeneous (even or odd). In view of the P.B.W. theorem for Lie superalgebras [24] it is easily demonstrated that a standard cyclic 
module $V=U v_{0}$ is indecomposable and that the normal form of Schur's lemma applies: i.e. if $T \in \operatorname{End} V$ is an $L$-invariant [20] then $T$ reduces to a scalar multiple of the identity on $V$. It is important to note that, unlike normal simple Lie algebras, finite dimensional standard cyclic modules may exist which are not irreducible.

Now let $Z$ denote the centre of the universal enveloping algebra $U$; i.e.

$$
Z=\{z \in U \mid z u=u z, \forall u \in U\} .
$$

It follows, from the remarks above, that if $V=U v_{0}$ is standard cyclic with highest weight $\lambda \in H^{*}$ then the elements of the centre $Z$ take constant values on $V$ (and this eigenvalue depends only on the highest weight $\lambda$ ). We denote the eigenvalue of $z \in Z$ on $V$ by $\chi_{\lambda}(z)$ and call $\chi_{\lambda}$ the infinitesimal character of $V$ : it determines an algebra homomorphism

$$
\chi_{\lambda}: Z \rightarrow \mathbf{C}, z \rightarrow \chi_{\lambda}(z) .
$$

More generally we say that a $U$-module $V$ admits the infinitesimal character $\chi_{\lambda}$, $\lambda \in H^{*}$, if the central elements $z \in Z$ take the constant value $\chi_{\lambda}(z)$ on $V$.

An important example of a central element is the universal Casimir element which may be constructed for basic classical Lie superalgebras in direct analogy with the normal Lie algebra case. To this end let $\left\{x_{1}, \ldots, x_{n}\right\}(n=\operatorname{dim} L)$ be a (homogeneous) basis for $L$ and let $\left\{x^{1}, x^{2}, \ldots, x^{n}\right\}$ be the corresponding left dual basis with respect to the invariant bilinear form (,) on $L$; viz.

$$
\left(x^{l}, x_{j}\right)=\delta_{j}^{l} \text {. }
$$

Then the operator

$$
C_{L}=\sum_{i=1}^{n} x_{i} x^{i}
$$

is easily seen to be a central element (see Appendix A) called the universal Casimir element. The eigenvalue of $C_{L}$ on a maximal weight state of weight $\lambda$ is easily seen to be given by (see Appendix $\mathrm{A}$ )

$$
\chi_{\lambda}\left(C_{L}\right)=(\lambda, \lambda+2 \delta)
$$

which is in direct analogy with Racah's well known formula for normal Lie algebras.

In conclusion we note that every finite dimensional irreducible $U$-module contains a (unique) maximal weight vector and hence is standard cyclic. As for Lie algebras a finite dimensional irreducible $U$-module is uniquely characterized by its highest weight [18]: we denote the finite dimensional irreducible $U$-module with highest weight $\lambda$ by $V(\lambda)$. It is important to note, however, that the finite dimensional irreducible $U$-module $V(\lambda)$ is not uniquely characterized by its infinitesimal character $\chi_{\lambda}$. That is there may exist finite dimensional irreducible 
$U$-modules $V(\lambda), V(\mu)$ with $\lambda \neq \mu$ but $\chi_{\lambda}=\chi_{\mu}$. Kac [18] calls $V(\lambda)$ typical if it is uniquely characterized, among the finite dimensional irreducible $U$-modules, by its infinitesimal character $\chi_{\lambda}$. Such finite dimensional irreducible $U$-modules necessarily split in all finite dimensional $U$-modules $[17,18]$. Typical modules, for a basic classical Lie super-algebra, have been characterized (in terms of their highest weights) by Kac [18]. Typical modules for the orthosymplectic Lie superalgebras have also been investigated, using Young diagram techniques, by Farmer and Jarvis [4].

\section{Polynomial identities}

Throughout we shall let

$$
V(\lambda)=V_{0}(\lambda) \oplus V_{1}(\lambda)
$$

be a fixed (but arbitrary) finite dimensional irreducible $U$-module with highest weight $\lambda$. Let $\left\{\lambda_{1}, \ldots, \lambda_{k}\right\}$ be the set of distinct weights in $V(\lambda)$ occurring with multiplicities $n_{1}, \ldots, n_{k}$ respectively.

Now $U$ may be imbedded in $U \otimes U$ by the diagonal homomorphism [24]

$$
d: U \rightarrow U \otimes U
$$

defined for $x \in L$ by

$$
d x=x \otimes 1+1 \otimes x
$$

which we extend to an algebra homomorphism to all of $U$. In general $d u$ for arbitrary $u \in U$ is a more complicated expression which may be written

$$
d u=\sum_{r} u_{r} \otimes v_{r}, \quad u_{r}, v_{r} \in U
$$

Now let $Y$ be the algebra

$$
Y=[\operatorname{End} V(\lambda)] \otimes U
$$

and let $\pi_{\lambda}$ be the representation afforded by $V(\lambda)$. Following refs [11, 19] we consider the map

$$
\partial: U \rightarrow Y
$$

defined for $x \in L$ by

$$
\partial(x)=\pi_{\lambda}(x) \otimes 1+1 \otimes x
$$

which we extend to an algebra homomorphism to all of $U$. More generally if $u \in U$ with $d u$ as in (3.1) we have

$$
\partial(u)=\sum_{r} \pi_{\lambda}\left(u_{r}\right) \otimes v_{r}
$$


In view of the canonical isomorphism

$$
Y \cong \operatorname{Hom}(V(\lambda), V(\lambda) \otimes U)
$$

we may regard elements of $Y$ as acting on $V(\lambda)$ according to

$$
(\rho \otimes u)(v)=(-1)^{\alpha \beta} \rho v \otimes u
$$

for all $v \in V_{\alpha}(\lambda), u \in U_{\beta}(\alpha, \beta=0,1)$ and $\rho \in \operatorname{End} V(\lambda)$, which we extend linearly to all of $Y$. By this means we may regard the elements of $Y$ as constituting $d \times d$ matrices $[d=\operatorname{dim} V(\lambda)]$ with entries from $U$. To be more explicit let $\left\{v_{1}, v_{2}, \ldots, v_{d}\right\}$ be a fixed homogeneous basis for $V(\lambda)$ and let $(j)$ denote the degree of index $j(=1, \ldots, d)$; i.e. $(j)=0$ (resp. 1) according to whether $v_{j} \in V_{0}(\lambda)$ [resp. $V_{1}(\lambda)$ ]. Then for $y \in Y$ we may write

$$
y v_{j}=\sum_{i=1}^{d} v_{i} \otimes y_{i j}, \quad y_{i j} \in U
$$

where $\left[y_{i j}\right.$ ] constitutes a $d \times d$ matrix. For example given homogeneous $x \in L$ we see from (3.2) that the matrix corresponding to $\partial(x) \in Y$ is given by

$$
\partial(x)_{i j}=(-1)^{(x)(j)} \delta_{i j} x+\pi_{\lambda}(x)_{i j} .
$$

More generally if $u \in U$ with $d u$ as in (3.1) we have

$$
\partial(u)_{\imath \jmath}=\sum_{r}(-1)^{\left(v_{r}\right)(j)} \pi_{\lambda}\left(u_{r}\right)_{\imath \jmath} v_{r}
$$

where $\left(v_{r}\right)$ denotes the degree of the universal enveloping algebra element $v_{r}$.

Throughout the remainder of this paper we let $z \in Z$ be a fixed (but arbitrary) element of $Z$. We let $A_{z} \in Y$ denote the operator

$$
A_{z}=-\frac{1}{2}\left[\partial(z)-\pi_{\lambda}(z) \otimes 1-1 \otimes z\right] .
$$

We choose this form since when $z=C_{L}$ we obtain

$$
A_{C_{L}}=-\frac{1}{2}\left[\partial\left(C_{L}\right)-\pi_{\lambda}\left(C_{L}\right) \otimes 1-1 \otimes C_{L}\right]
$$

which, in view of (2.6), may be alternatively written

$$
A_{C_{L}}=-\sum_{a} \pi_{\lambda}\left(x_{a}\right) \otimes x^{a}=-\sum_{a}(-1)^{(a)} \pi_{\lambda}\left(x^{a}\right) \otimes x_{a},
$$

where $(a)$ denotes the degree of index $a(=1, \ldots, n)$. For simplicity we denote the matrix of (3.5) and (3.6) simply by $A_{L}$. In view of equ. (3.3) we clearly have

$$
\left[A_{L}\right]_{i j}=-\sum_{a}(-1)^{(a)(j)} \pi_{\lambda}\left(x_{a}\right)_{i j} x^{a}
$$

which is a matrix with entries from $L$.

In the special case that $\pi_{\lambda}$ corresponds to the contragredient vector representation, the matrix of (3.7) reduces to the matrix considered by Jarvis and Green [14] for the Lie superalgebras $g l(n, m)$ and $\operatorname{osp}(n, m)$. In such a case it can be shown 
[14] that, on a finite dimensional irreducible representation of $g l(n, m)$ or $\operatorname{osp}(n, m)$, the matrix of (3.7) satisfies a polynomial identity of degree $(n+m)$. It is our aim here to extend this result to the more general case where $\pi_{\lambda}$ is any finite dimensional irreducible representation for a basic classical Lie superalgebra $L$ and $z \in Z$ is arbitrary. Our main result is

THEOREM 1. Let $V=U v_{0}$ be a standard cyclic module ( finite or infinite dimensional) with highest weight $\mu \in H^{*}$. Then on $V$ the matrix $A_{z}$ satisfies the polynomial identity

$$
\prod_{i=1}^{k}\left(A_{z}-f_{z, i}(\mu)\right)=0,
$$

where $f_{z, i}$ denotes the polynomial function

$$
f_{z, i}(\mu)=-\frac{1}{2}\left[\chi_{\mu+\lambda_{1}}(z)-\chi_{\lambda}(z)-\chi_{\mu}(z)\right], \quad i=1, \ldots, k .
$$

Throughout we assume that $V=U v_{0}$ is a standard cyclic module where $v_{0}$ is a highest weight vector of weight $\mu \in H^{*}$, and we let $\pi$ denote the representation afforded by $V$. We proceed by determining the possible infinitesimal characters which occur in the tensor product space $V(\lambda) \otimes V$. We require first the following elementary result:

LEMMA 1. Let $v_{-}^{\lambda}$ denote the minimal weight vector of $V(\lambda)$. Then $V(\lambda) \otimes V$ is cyclically generated by the vector $v_{-}^{\lambda} \otimes v_{0}$; i.e.

$$
V(\lambda) \otimes V=U\left(v_{-}^{\lambda} \otimes v_{0}\right) .
$$

Proof. Set $W=U\left(v_{-}^{\lambda} \otimes v_{0}\right)$ and define

$$
V^{\prime}=\{v \in V \mid V(\lambda) \otimes v \subseteq W\} .
$$

Clearly $V^{\prime}$ is a $U$ - submodule of $V$ since for $v \in V^{\prime}$ and homogeneous $x \in L$

$$
(-1)^{\alpha(x)} w \otimes x v=x(w \otimes v)-(x w) \otimes v \in W
$$

(for all $w \in V_{\alpha}(\lambda)$ ), which implies $x v \in V^{\prime}$ for all $x \in L$. To prove the lemma it suffices to show $V^{\prime}=V$ and for this it suffices to demonstrate, by the cyclic nature of $v_{0} \in V$, that $v_{0} \in V^{\prime}$.

We have, by the P.B.W. theorem [notation as in (2.3)]

$$
V(\lambda)=U v_{-}^{\lambda}=U(B) U\left(N^{-}\right) v_{-}^{\lambda}=U(B) v_{-}^{\lambda},
$$


where $U(B)$ [resp. $U\left(N^{-}\right)$] denotes the universal enveloping algebra of $B$ (resp. $\left.N^{-}\right)$. Thus we obtain

$$
U(B)\left(v_{-}^{\lambda} \otimes v_{0}\right)=\left[U(B) v_{-}^{\lambda}\right] \otimes v_{0}=V(\lambda) \otimes v_{0},
$$

which shows that $V(\lambda) \otimes v_{0} \subseteq W$ and hence $v_{0} \in V^{\prime}$ as required. Q.E.D.

We now assume that the distinct weights $\lambda_{1}, \lambda_{2}, \ldots, \lambda_{k}$ are ordered in non-decreasing order with respect to the partial ordering $>$ induced by the positive roots; i.e. if $\lambda_{i}>\lambda_{j}$ then $i>j$. We denote the weight space of $V(\lambda)$ consisting of vectors of weight $\lambda_{t}$ by $V_{t}(\lambda)\left[n_{t}=\operatorname{dim} V_{i}(\lambda)\right]$ and we set

$$
\begin{gathered}
S_{i}=\bigoplus_{j=i}^{k} V_{i}(\lambda), \\
\tilde{S}_{i}=S_{l} \otimes v_{0} .
\end{gathered}
$$

By our construction each space $S_{i}$ (and hence $\tilde{S}_{i}$ ) is stable under the action of $B$ and we have the following descending chain of $U(B)$ modules:

$$
V(\lambda) \otimes v_{0}=\tilde{S}_{1} \supset \tilde{S}_{2} \supset \cdots \tilde{S}_{k} \supset \tilde{S}_{k+1}=(0) .
$$

Applying $U$ to the left of this chain we obtain the descending chain of $U$-modules:

$$
W_{1} \supseteq W_{2} \supseteq \cdots W_{k} \supseteq W_{k+1}=(0),
$$

where $W_{\imath}=U \tilde{S}_{\imath}$. We observe, from Lemma 1, that

$$
W_{1}=U\left(V(\lambda) \otimes v_{0}\right)=V(\lambda) \otimes V .
$$

We have the following result (notation as above):

LEMMA 2. Each factor module $M_{i}=W_{i} / W_{t+1}(i=1, \ldots, k)$ is either $(0)$ or else admits the infinitesimal character $\chi_{\mu+\lambda_{1}}$. In particular the infinitesimal characters occurring in $V(\lambda) \otimes V$ are of the form $\chi_{\mu+\lambda_{i}}(i=1, \ldots, k)$.

Proof. We note that since each weight space $V_{1}(\lambda)$ is $\mathbf{Z}_{2}$-graded [i.e. $v \in V_{1}(\lambda)$ implies the even and odd parts of $v$ belong to $\left.V_{i}(\lambda)\right]$ the $U$-modules $W_{i}$ of (3.10) are graded as required. By our construction we have

$$
\tilde{S}_{t}=V_{t}(\lambda) \otimes v_{0} \oplus \tilde{S}_{t+1}
$$

and hence

$$
W_{\imath}=U\left[V_{\imath}(\lambda) \otimes v_{0}\right]+W_{i+1} .
$$

Thus we may write (isomorphism theorem)

$$
\begin{aligned}
M_{i} & =\left(U\left[V_{i}(\lambda) \otimes v_{0}\right]+W_{i+1}\right) / W_{i+1} \\
& \cong U\left[V_{i}(\lambda) \otimes v_{0}\right] / W_{i+1} \cap U\left[V_{i}(\lambda) \otimes v_{0}\right] .
\end{aligned}
$$

Now observe that [notation as in (2.3)]

$$
N^{+}\left[V_{i}(\lambda) \otimes v_{0}\right] \subseteq \tilde{S}_{t+1} \subseteq W_{t+1}
$$


and hence

$$
N^{+}\left[V_{i}(\lambda) \otimes v_{0}\right] \subseteq W_{i+1} \cap U\left[V_{i}(\lambda) \otimes v_{0}\right],
$$

from which it follows that $M_{1}$ in (3.11) is either (0) or else a sum (not necessarily direct) of standard cyclic modules of highest weight $\mu+\lambda_{i}$. Indeed if $\left\{w_{1}, \ldots, w_{r}\right\}$ $\left(r=n_{i}\right)$ denotes a homogeneous basis for $V_{i}(\lambda) \otimes v_{0}$ we have

$$
M_{i} \cong \sum_{j=1}^{r} U \bar{w}_{j}
$$

where $\bar{w}_{j}$ denotes the left coset

$$
\bar{w}_{j}=w_{j}+W_{i+1} \cap U\left[V_{i}(\lambda) \otimes v_{0}\right] .
$$

Clearly each module $U \bar{w}_{j}$, if non zero, is standard cyclic since $N^{+} \bar{w}_{j}=(0)$. It follows in particular that if $M_{i} \neq(0)$ then $M_{i}$ necessarily admits the infinitesimal character $\chi_{\mu+\lambda_{i}}$. Q.E.D.

Corollary (notation as above). Suppose $V=U v_{0}$ is a finite dimensional standard cyclic module and $\mu+\lambda_{1}$ is a typical dominant weight. Then the irreducible module $V\left(\mu+\lambda_{i}\right)$ occurs in $V(\lambda) \otimes V$ with multiplicity less than or equal to $n_{i}$.

ProOF. In such a case we note that the standard cyclic modules occurring in the decomposition (3.12) are either (0) or else irreducible [18]. In particular $M_{i}$ is a direct sum of at most $n_{t}$ summands $V\left(\mu+\lambda_{l}\right)$ and splits in $V(\lambda) \otimes V$. We note also, by the definition of typical module, that $V\left(\mu+\lambda_{1}\right)$ cannot occur in $M_{\mathrm{J}}$ for $j \neq i$. This completes the proof. Q.E.D.

We are now in a position to set up the last step in the proof of Theorem 1 . From (3.4) we see that, acting on the $U$-module $V$, the matrix $A_{z}$ may be written

$$
A_{z}=-\frac{1}{2}\left[\pi_{\lambda} \otimes \pi(z)-\pi_{\lambda}(z) \otimes 1-1 \otimes \pi(z)\right] .
$$

It follows from Lemma 2 that on each factor module $M_{i}=W_{i} / W_{i+1}$ of the chain (3.10) the operator $A_{z}$ takes the constant value

$$
f_{z, i}(\mu)=-\frac{1}{2}\left[\chi_{\mu+\lambda_{i}}(z)-\chi_{\lambda}(z)-\chi_{\mu}(z)\right] \text {. }
$$

These are clearly the generalized eigenvalues of $A_{z}$ on the space $V(\lambda) \otimes V$ and it follows immediately that $A_{z}$ satisfies the polynomial identity of (3.8). This completes the proof of the theorem.

REMARKS. In order to clarify the two ways of viewing $A_{z}$, either as an operator on $V(\lambda) \otimes V$ or else a $d \times d$ matrix over $U$ whose entries are operators on $V$, we have

$$
A_{z}\left(v_{j} \otimes v\right)=\sum_{i} v_{i} \otimes\left[A_{z}\right]_{i j} v, \quad \forall v \in V
$$


More generally if $p(x)$ is any polynomial with invariant coefficients we have

$$
p\left(A_{z}\right)(v, \otimes v)=\sum_{i} v_{i} \otimes p\left(A_{z}\right)_{i j} v, \quad v \in V
$$

where polynomials in the matrix $A_{z}$ are given recursively according to

$$
\left(A_{z}^{m+1}\right)_{i j}=\sum_{k}\left(A_{z}\right)_{i k}\left(A_{z}^{m}\right)_{k j}=\sum_{k}\left(A_{z}^{m}\right)_{i k}\left(A_{z}\right)_{k j} .
$$

Thus we have $p\left(A_{z}\right)=0$ on $V(\lambda) \otimes V$ if and only if $p\left(A_{z}\right)_{i j}=0$ on $V(i, j=$ $1, \ldots, d)$. Hence the identity of $(3.8)$ is equivalent to the following $d^{2}$ identities which must hold on $V$ :

$$
\prod_{i=1}^{k}\left[A_{z}-f_{z, 1}(\mu)\right]_{p q}=0 ; \quad p, q=1, \ldots, d .
$$

As a special case of Theorem 1 we note that, acting on a standard cyclic module of highest weight $\mu$, the matrix $A_{L}$ of (3.7) satisfies the polynomial identity

$$
\prod_{i=1}^{k}\left[A_{L}-\frac{1}{2}(\lambda, \lambda+2 \delta)+\frac{1}{2}\left(\lambda_{i}, \lambda_{i}+2(\mu+\delta)\right)\right]=0
$$

where we have used the result [c.f. (3.9)]

$$
f_{C_{L}, i}(\mu)=\frac{1}{2}(\lambda, \lambda+2 \delta)-\frac{1}{2}\left(\lambda_{i}, \lambda_{i}+2(\mu+\delta)\right)
$$

which follows immediately from (2.7). The identities of (3.13) include the identities of Jarvis and Green [14] as a special case. Identities of the more general type (3.8) have been previously discussed by Jarvis and Murray [15]. In this latter work it was pointed out (on the basis of some unpublished work of the present author) that the matrix $A_{z}$ satisfies a polynomial identity whose factors could be put in 1-1 correspondence with the weights (including multiplicities) of $V(\lambda)$. In particular the matrix $A_{z}$ satisfies a polynomial identity of degree $d=\operatorname{dim} V(\lambda)$. Theorem 1 explicity demonstrates the stronger result that the factors of the characteristic identity satisfied by the matrix $A_{z}$ may be put in 1-1 correspondence with the distinct weights in $V(\lambda)$.

\section{The adjoint matrix}

We note that associated with the matrix $A_{z}$ is its adjoint $\overline{A_{z}}$ which is obtained by following exactly the same procedure as before but with $\pi_{\lambda}$ replaced by its dual $\pi_{\lambda}^{*}$. It can be shown that

$$
\left[\overline{A_{z}}\right]_{i j}=(-1)^{(i)+(i)(j)} \theta \circ\left[A_{\bar{z}}\right]_{j i}
$$


where $\theta$ denotes the principal anti-automorphism of $U[24]$ and $\bar{z}=\theta(z)$. Thus for example the adjoint of the matrix $A_{L}$ of (3.7) is given by

$$
\begin{aligned}
{\left[\bar{A}_{L}\right]_{i j} } & =-\sum_{a}(-1)^{(a)(J)} \pi_{\lambda}^{*}\left(x_{a}\right)_{i j} x^{a} \\
& =\sum_{a}(-1)^{a} \pi_{\lambda}\left(x_{a}\right)_{j l} x^{a}=\sum_{a} \pi_{\lambda}\left(x^{a}\right)_{j l} x_{a} .
\end{aligned}
$$

In the case that $\pi_{\lambda}$ corresponds to the fundamental vector representation of the Lie superalgebras $g l(n, m)$ or $\operatorname{osp}(n, m)$ this is the matrix considered by Jarvis and Green [14].

It follows immediately from Theorem 1 that, on a standard cyclic module with highest weight $\mu \in H^{*}$, the matrix $\overline{A_{z}}$ satisfies the polynomial identity

$$
\prod_{i=1}^{k}\left(\overline{A_{z}}-\bar{f}_{z, l}(\mu)\right)=0
$$

where $\left[\lambda^{*}\right.$ the highest weight of $\left.V(\lambda)^{*}\right]$

$$
\bar{f}_{z, i}(\mu)=-\frac{1}{2}\left[\chi_{\mu-\lambda_{t}}(z)-\chi_{\lambda^{*}}(z)-\chi_{\mu}(z)\right]
$$

where we have used the result that the weights occurring in $V(\lambda)^{*}$ are the negative of those occurring in $V(\lambda)$. We note that

$$
\chi_{\lambda^{*}}(z)=\chi_{\lambda}(\bar{z}), \bar{z}=\theta(z)
$$

Hence, in the special case that $z=C_{L}$, we have $C_{L}=\bar{C}_{L}$ from which it follows that $\chi_{\lambda}\left(C_{L}\right)=\chi_{\lambda^{*}}\left(C_{L}\right)$. In such a case the polynomial functions of (4.2) reduce to

$$
\bar{f}_{C_{L, t}}(\mu)=\frac{1}{2}(\lambda, \lambda+2 \delta)-\frac{1}{2}\left(\lambda_{i}, 2(\mu+\delta)-\lambda_{i}\right) .
$$

Hence, as a special case of (4.1) we see that the adjoint matrix $\overline{A_{L}}$ satisfies the polynomial identity

$$
\prod_{i=1}^{k}\left[\overline{A_{L}}-\frac{1}{2}(\lambda, \lambda+2 \delta)+\frac{1}{2}\left(\lambda_{l}, 2(\mu+\delta)-\lambda_{\imath}\right)\right]=0
$$

on a standard cyclic module of highest weight $\mu \in H^{*}$.

Note that, as well as an adjoint, the matrix $A_{z}$ also possesses a double and triple adjoint $\overline{\overline{A_{z}}}$ and $\overline{\overline{A_{z}}}$ respectively. We have

$$
\left[\overline{\overline{A_{z}}}\right]_{i j}=\gamma \circ\left[A_{z}\right]_{i j},\left[\overline{\overline{A_{z}}}\right]_{i j}=\gamma \circ\left[\overline{A_{z}}\right]_{i j}
$$

where $\gamma$ denotes the grading automorphism on $U[24]$. The matrix $\overline{\overline{A_{z}}}$ (resp. $\overline{\overline{A_{z}}}$ ) satisfies the same polynomial identity as the matrix $A_{z}$ (resp. $\overline{A_{z}}$ ).

\section{Generalizations}

In the previous sections polynomial identities were derived, in arbitrary representations generated by a maximal (or minimal) weight vector, for the matrices of (3.4). Throughout it was assumed that $\pi_{\lambda}$ was a finite dimensional irreducible 
representation with highest weight $\lambda$. However, in applications one frequently has to deal with indecomposable finite dimensional representations. Accordingly we conclude in this section by generalizing our previous results to the case where $\pi_{\lambda}$ is a finite dimensional indecomposable (not necessarily irreducible) representation.

We remark that a finite dimensional module over a basic classical Lie superalgebra $L$ necessarily decomposes into a unique (up to isomorphism and ordering) direct sum of indecomposable submodules. Moreover every finite dimensional indecomposable $L$-module $V$ is necessarily primary: that is $V$ admits a composition series whose factors all necessarily admit the same infinitesimal character, $\chi_{\lambda}$ say. We call $\chi_{\lambda}$ the characteristic of the indecomposable module $V$. Note that if $I_{\lambda} \subset Z$ is the kernel of the infinitesimal character $\chi_{\lambda}$ (viz. $I_{\lambda}=\left\{z \in Z \mid \chi_{\lambda}(z)=\right.$ $0\}$ ) then $I_{\lambda}$ constitutes a maximal two-sided ideal in $Z$ of co-dimension 1 (c.f. ref. $[10,19])$. Thus if $V$ is indecomposable and finite dimensional with characteristic $\chi_{\lambda}$, then there exists $m \in \mathbf{Z}^{+}$such that $I_{\lambda}^{m} V=(0)$. The smallest such positive integer is called the characteristic length of the $L$-module $V$.

Hence, throughout the remainder of this paper, we assume that $V(\lambda)$ is a finite dimensional indecomposable $L$-module with characteristic $\chi_{\lambda}$ and we let $\pi_{\lambda}$ be the representation afforded by $V(\lambda)$. Following our previous notation we set

$$
A_{z}=-\frac{1}{2}\left[\partial(z)-\pi_{\lambda}(z) \otimes 1-1 \otimes z\right]
$$

where $z \in Z$, with $\partial: U \rightarrow Y$ as before. We let $\left\{\lambda_{1}, \ldots, \lambda_{k}\right\}$ denote the set of distinct weights in $V(\lambda)$ which we assume, as before, are in non-decreasing order with respect to the partial ordering $>$ induced by the positive roots. It is our aim here to show that Theorem 1 holds also for the more general matrices of (5.1).

We follow exactly the same construction as before and consider the descending chain of $U$-modules (3.10). It is easily seen that our previous method of proof goes over, unchanged, to the more general case we are considering provided we can demonstrate the result

$$
V(\lambda) \otimes V=W_{1}
$$

where

$$
W_{1}=U\left[V(\lambda) \otimes v_{0}\right]
$$

To this end consider the subspace of $V$ defined by

$$
V^{\prime}=\left\{v \in V \mid V(\lambda) \otimes v \subseteq W_{1}\right\} .
$$

To prove (5.2) it clearly suffices to demonstrate that $V^{\prime}=V$. Using the same method of proof as that used in Lemma 1 , it is easily seen that $V^{\prime}$ is a (graded) submodule of $V$ which, by our construction, necessarily contains the vector $v_{0}$. The cyclicity of the vector $v_{0}$ then forces $V^{\prime}=V$ and the result is seen to follow. 
Thus we have established that the more general matrices of (5.1), corresponding to indecomposable $\pi_{\lambda}$, also satisfy the polynomial identities of Theorem 1. In particular the polynomial identities (3.13), which include the identities of Jarvis and Green [14], also extend to indecomposable (reference) representations $\pi_{\lambda}$.

We remark that the polynomial identities of Theorem 1 need not be the minimum polynomial identities satisfied by the matrices of (5.1). We therefore conclude by obtaining the minimal polynomial identities along the lines suggested in $[10]$.

We have demonstrated that the infinitesimal characters occurring in the tensor product module $V(\lambda) \otimes V$ (notation as above) are necessarily of the form $\chi_{\mu+\lambda_{i}}(i=1, \ldots, k)$. Although the weights

$$
\lambda_{1}, \lambda_{2}, \ldots, \lambda_{k}
$$

are all distinct it is not necessarily true $[17,18]$ that the infinitesimal characters

$$
\chi_{\mu+\lambda_{1}}, \chi_{\mu+\lambda_{2}}, \ldots, \chi_{\mu+\lambda_{k}}
$$

are distinct. Assume the number of distinct ones is $n(\leqslant k)$ and suppose the weights are renumbered so that the infinitesimal characters $\chi_{\mu+\lambda_{1}}(i=1, \ldots, n)$ are all distinct. For $i=1, \ldots, n$ let $m_{i}$ be the multiplicity of the infinitesimal character $\chi_{\mu+\lambda}$ in the sequence (5.3). With this notation the polynomial identity of Theorem 1 may be written

$$
\prod_{i=1}^{n}\left(A_{z}-f_{z, i}(\mu)\right)^{m_{\imath}}=0 .
$$

From these identities it is an easy matter to deduce $[10,19]$ that the space $X=V(\lambda) \otimes V$ decomposes into a direct sum of primary submodules

$$
X=\bigoplus_{i=1}^{n} X_{i}
$$

where (notation as above)

$$
X_{i}=\left\{v \in X \mid I_{\mu+\lambda_{t}}^{m} v=(0)\right\}
$$

is the unique maximal submodule of $X$ with characteristic $\chi_{\mu+\lambda_{i}}$. It follows therefore, that if $z \in Z$ is such that the numbers $\chi_{\mu+\lambda_{1}}(z)(i=1, \ldots, n)$ are distinct, then the minimum polynomial identity satisfied by $A_{z}$ on $V$ is given by

$$
\prod_{i=1}^{n}\left(A_{z}-f_{z, i}(\mu)\right)^{n_{i}}=0,
$$

where $n_{i}\left(\leqslant m_{i}\right)$ is the characteristic length of the primary module $X_{i}$. We see from this that a knowledge of the minimal polynomial identities is equivalent to a knowledge of the characteristic lengths of the primary modules occurring in the decomposition (5.5). Finally we note that projection operators which project onto 
the primary submodules $X_{i}$ may be explicitly constructed using the polynomial identities (5.4) as suggested in [10]. Such projection operators are useful for projecting out shift components of (super) tensor operators [10].

\section{Acknowledgements}

The author would like to thank Dr. P. D. Jarvis and Dr. S. A. Edwards for several useful discussions. The author also acknowledges the finanical support of an A.R.G.S. research grant.

\section{Appendix A: The Casimir invariant}

Let $\left\{x_{a}\right\}_{a-1}^{n}$ be a homogeneous basis for $L$ and let $(a)$ be the degree of index $a$. We then have the metric tensor

$$
g_{a b}=\left(x_{a}, x_{b}\right)=(-1)^{(a)(b)}\left(x_{b}, x_{a}\right),
$$

where (,) is the fixed invariant bilinear form on $L$. We note that the form (,) is even and hence $g_{a b}=0$ unless $(a)=(b)$. Thus we may write

$$
g_{a b}=(-1)^{(a)} g_{b a}=(-1)^{b} g_{b a} .
$$

Since the form (,) is non-degenerate we may introduce the inverse metric $g^{a b}$; i.e.

$$
g^{a b} g_{b c}=g_{c b} g^{b a}=\delta_{c}^{a} .
$$

The left dual basis to $\left\{x_{a}\right\}_{a-1}^{n}$ is then given by

$$
x^{a}=g^{a b} x_{b}
$$

which satisfies

$$
\left(x^{a}, x_{b}\right)=\delta_{b}^{a}
$$

Note however that

$$
\left(x_{b}, x^{a}\right)=(-1)^{(a)} \delta_{b}^{a}
$$

so that $x^{a}$ is not a right dual basis. It is our aim to show that

$$
C_{L}=\sum_{a} x_{a} x^{a}=\sum_{a}(-1)^{(a)} x^{a} x_{a}
$$

indeed constitutes a Casimir invariant; i.e.

$$
x_{b} C_{L}-C_{L} x_{b}=0 ; b=1, \ldots, n .
$$

We write the graded commutation relations in the form

$$
\left[x_{a}, x_{b}\right]=\Gamma_{a b}^{c} x_{c}
$$


where the structure constants $\Gamma_{a b}^{c}$ satisfy

$$
\Gamma_{a b}^{c}=-(-1)^{(a)(b)} \Gamma_{b a}^{c} \text {. }
$$

We also have

$$
\begin{aligned}
g^{a c} \Gamma_{c b}^{p} g_{p d} & =\left(\left[x^{a}, x_{b}\right], x_{d}\right) \\
& =\left(x^{a},\left[x_{b}, x_{d}\right]\right) \\
& =\Gamma_{b d}^{a},
\end{aligned}
$$

which follows from the invariance of the form (,). Equations $\left(A_{1}\right)$ and $\left(A_{2}\right)$ together imply the symmetry relation

$$
\begin{aligned}
g^{a c} \Gamma_{c b}^{p} & =g^{c p} \Gamma_{b c}^{a} \\
& =-(-1)^{(\gamma)((\gamma)+(\beta))} g^{p c} \Gamma_{c b}^{a} .
\end{aligned}
$$

We have

$$
\begin{aligned}
C_{L} x_{b}-x_{b} C_{L} & =\left(x_{a} x^{a}\right) x_{b}-x_{b}\left(x_{a} x^{a}\right) \\
& =x_{a}\left[x^{a}, x_{b}\right]+(-1)^{(a)(b)}\left[x_{a}, x_{b}\right] x^{a}
\end{aligned}
$$

where [,] is the graded bracket operation on $L$. We thus obtain

$$
\begin{aligned}
C_{L} x_{b}-x_{b} C_{L} & =x_{a} g^{a c}\left[x_{c}, x_{b}\right]+(-1)^{(a)(b) g^{a c}}\left[x_{a}, x_{b}\right] x_{c} \\
& =\left(g^{d a} \Gamma_{a b}^{c}-g^{a c} \Gamma_{b a}^{d}\right) x_{d} x_{c} \\
& =0
\end{aligned}
$$

as required, where we have applied the symmetry relation $\left(\mathrm{A}_{3}\right)$.

To determine the eigenvalues of $C_{L}$ we note that $C_{L}$ is independent of the basis chosen for $L$. We therefore choose positive root vectors $x_{\alpha} \in L_{\alpha}\left(\alpha \in \Phi^{+}\right)$and we let $\left\{x^{\alpha}\right\}_{\alpha>0}$ be the corresponding left dual vectors; ie

$$
\left(x^{\alpha}, x_{\beta}\right)=\delta_{\beta}^{\alpha}, \quad x^{\alpha} \in L_{-\alpha} .
$$

From Kac [18] we note that if $x_{\alpha} \in L_{\alpha}, x_{-\alpha} \in L_{-\alpha}$ then

$$
\left[x_{\alpha}, x_{-\alpha}\right]=\left(x_{\alpha}, x_{-\alpha}\right) h_{\alpha} \text {, }
$$

where $h_{\alpha}$ is defined by

$$
\left(h_{\alpha}, h\right)=\alpha(h), \quad h \in H .
$$

We therefore have, in particular,

$$
\left[x^{\alpha}, x_{\alpha}\right]=\left(x^{\alpha}, x_{\alpha}\right) h_{-\alpha}=-h_{\alpha} .
$$

At this point we distinguish between even and odd roots and choose as a basis for $L$

$$
\begin{array}{ll}
x_{\alpha}, x^{\alpha}, & \alpha \in \Phi_{0}^{+}, \\
x_{\alpha}, x^{\alpha}, & \alpha \in \Phi_{1}^{+}, \\
h_{i}, & i=1, \ldots, l,
\end{array}
$$


where $\left\{h_{i}\right\}_{i=1}^{\prime}$ constitutes a basis for $H$. The corresponding left dual basis to $\left(\mathrm{A}_{6}\right)$ is given by

$$
\begin{array}{ll}
x^{\alpha}, x_{\alpha}, & \alpha \in \Phi_{0}^{+}, \\
x^{\alpha},-x_{\alpha}, & \alpha \in \Phi_{1}^{+}, \\
h^{i}, & i=1, \ldots, l .
\end{array}
$$

We note the following commutation relations

$$
\begin{array}{ll}
{\left[x_{\alpha}, x^{\alpha}\right]=h_{\alpha},} & \alpha \in \Phi_{0}^{+}, \\
{\left[x_{\alpha}, x^{\alpha}\right]=-h_{\alpha},} & \alpha \in \Phi_{1}^{+} .
\end{array}
$$

We note also that if $\lambda \in H^{*}$ then we have an expansion

$$
h_{\lambda}=\sum_{i} \lambda\left(h_{\imath}\right) h^{i}
$$

where $h_{\lambda}$ is given by equ. (2.5). It follows therefore, that for $\lambda, \mu \in H^{*}$, we may write

$$
(\lambda, \mu)=\left(h_{\lambda}, h_{\mu}\right)=\sum_{i} \lambda\left(h_{i}\right) \mu\left(h^{i}\right) .
$$

In the basis of $\left(\mathrm{A}_{6}\right)$ the universal Casimir invariant $C_{L}$ may be written

$$
C_{L}=\sum_{\alpha \in \Phi_{0}^{+}}\left(x_{\alpha} x^{\alpha}+x^{\alpha} x_{\alpha}\right)+\sum_{\alpha \in \Phi_{1}^{+}}\left(x_{\alpha} x^{\alpha}-x^{\alpha} x_{\alpha}\right)+\sum_{l} h_{t} h^{i}
$$

Applying $C_{L}$ to a maximal weight state $v_{0}^{\lambda}$ of weight $\lambda \in H^{*}$ we have

$$
\chi_{\lambda}\left(C_{L}\right) v_{0}^{\lambda}=\sum_{\alpha \in \Phi_{0}^{+}} x_{\alpha} x^{\alpha} v_{0}^{\lambda}+\sum_{\alpha \in \Phi_{1}^{+}} x_{\alpha} x^{\alpha} v_{0}^{\lambda}+\sum_{i} \lambda\left(h_{i}\right) \lambda\left(h^{i}\right) v_{0}^{\lambda}
$$

where we have used $x_{\alpha} v_{0}^{\lambda}=0$ for $\alpha \in \Phi^{+}$. Applying the commutation relations $\left(A_{7}\right)$ and eq. $\left(A_{8}\right)$ we thereby obtain

$$
\begin{aligned}
\chi_{\lambda}\left(C_{L}\right) & =\lambda\left(h_{2 \delta_{0}}\right)-\lambda\left(h_{2 \delta_{1}}\right)+(\lambda, \lambda) \\
& =(\lambda, \lambda+2 \delta) .
\end{aligned}
$$

Finally, we remark that although the Casimir invariant $C_{L}$ is independent of the basis chosen for $L$ it is clear that $C_{L}$ and its eigenvalues depend (up to a scalar) on the invariant bi-linear form (, ) chosen for $L$.

\section{References}

[1] A. J. Bracken and H. S. Green, J. Math. Phys. 12 (1971), 2099.

[2] L. Corwin, Y. Ne'eman and S. Sternberg, Rev. Modern Phys. 47 (1975), 573.

[3] J. Dixmier, Enveloping algebras (North Holland, Amsterdam-New York-Oxford 1977).

[4] R. J. Farmer and P. D. Jarvis, "Representations of Orthosympectic Superalgebras 11. Young Diagrams and Weight Space Techniques", (Univ. of Tasmania preprint, 1983). 
[5] P. Fayet and S. Ferrara, Phys. Rep. C 32 (1977), 69.

[6] M. D. Gould, J. Math. Phys. 21 (1980), 444.

[7] M. D. Gould, J. Math. Phys. 22 (1981), 2376.

[8] M. D. Gould and G. S. Chandler, Int. J. Quantum Chem. 25 (1984), 553.

[9] M. D. Gould and G. S. Chandler, Int. J. Quantum Chem. 25 (1984), 603.

[10] M. D. Gould, J. Phys. A 17 (1984), 1.

[11] M. D. Gould, J. Austral. Math. Soc. Ser. B 26 (1984), 257.

[12] H. S. Green, J. Math. Phys. 12 (1971), 2106.

[13] J. E. Humphreys, Introduction to Lie algebras and representation theory (Springer-Verlag, New York-Heidelberg-Berlin, 1972).

[14] P. D. Jarvis and H. S. Green, J. Math. Phys. 20 (1979), 2115.

[15] P. D. Jarvis and M. K. Murray, J. Math. Phys. 24 (1983), 1705.

[16] V. G. Kac, Adv. in Math. 26 (1977), 8.

[17] V. G. Kac, Comm. Algebra 5 (1977), 889.

[18] V. G. Kac, Lecture Notes in Math. 676 (Springer, Berlin, 1978), 597.

[19] B. Kostant, J. Funct. Anal. 20 (1975), 257.

[20] W. Nahm and M. Scheunert, J. Math. Phys. 17 (1976), 868.

[21] A. Salam and J. Strathdee, Nuclear Phys. B 76 (1974), 477.

[22] J. Scherk, Rev. Modern Phys. 47 (1975), 123.

[23] M. Scheunert, W. Nahm and V. Rittenberg, J. Math. Phys. 17 (1976), 1626.

[24] M. Scheunert, Lecture Notes in Math. 716 (Springer, Berlin, 1979).

[25] J. Wess and B. Zumino, Nuclear Phys. B 70 (1974), 39. 